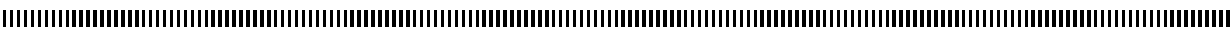

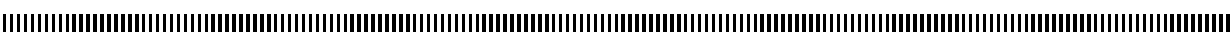
| | |

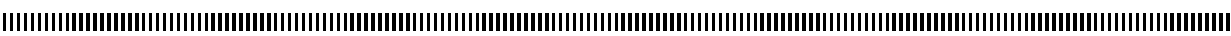

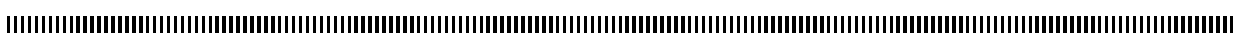

\title{
Two simple growth models in the chemostat
}

\author{
Jean-Luc Gouzé*a — Valérie Lemesle ${ }^{\mathrm{a}}$ \\ a COMORE, INRIA \\ BP 93, 06902 Sophia-Antipolis, \\ FRANCE \\ * Corresponding author \\ gouze@sophia.inria.fr
}

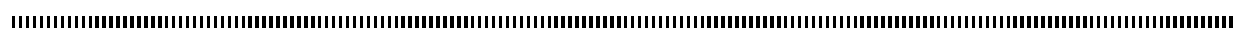

ABSTRACT. In a chemostat, transient oscillations are often experimentally observed during cell growth. The aim of this paper is to propose simple autonomous models which are able (or not) to generate these oscillations, and to investigate them analytically. Our point of view is based on a simplification of the cell cycle in which there are two states (mature and immature) with the transfer between the two dependent on the available resources. We built two similar models, one with cell biomass and the other with cell number density. We prove that the first one oscillates, but not the second. This paper is dedicated to Claude Lobry, who helped us to build a first version of these models.

RÉSUMÉ. On observe parfois des oscillations pendant la croissance de cellules dans un chémostat. Nous proposons ici deux modèles autonomes simples de croissance cellulaire (en dimension trois) basés sur des hypothèses réalistes, structurés en stades (les cellules matures et les cellules immatures) : le premier modèle est écrit en nombre de cellules, et peut présenter des oscillations. Le deuxième modèle est écrit en biomasse, et n'admet pas d'oscillations à cause de la conservation de la masse. Nous étudions les deux modèles. Cet article est dédié à Claude Lobry, qui a participé aux premières étapes de l'écriture des modèles.

KEYWORDS : chemostat, cell growth, oscillations

MOTS-CLÉS : chémostat, croissance cellulaire, oscillations

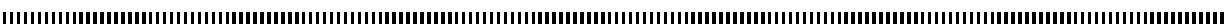




\section{Introduction}

A chemostat is a laboratory apparatus, composed of a reservoir fed by a constant liquid flow, used for experiments of controlled growth of micro-organisms. The inflow feeds the culture with biochemicals called substrates (e.g. nitrate). In the vessel, microorganisms grow consuming these nutrients, then the outflow retrieves substrates and cells present in the reservoir. Usually, only one substrate is limiting in order to evaluate its influence on cell growth. This idealized and controlled apparatus allows reproducible experiments and gives a very good approximation of specific realistic biological mechanisms (see for example [Sciandra et al.(2003)]).

Unstructured mathematical models, meaning that only one global variable is used to represent the microorganisms, are often employed to describe cell growth in a chemostat. The most representative one has been proposed by [Monod(1942)]. His approach was based on the interaction between microorganisms, more precisely bacteria, and substrates dissolved in the liquid medium. Although his model was successfully used to fit data $[\operatorname{Monod}(1942)]$, its predictions in perturbed conditions are far from satisfactory. In particular considering Saccharomyces cervisae [Porro et al.(1988)] or phytoplankton cells (e.g. Chlamydomonas reinhardii, [Nisbet and Gurney(1982)]), it is not able to represent oscillatory transients in cell number as observed in chemostat experiments (see section 2.2). A new modeling approach is therefore required.

One idea is to consider different uptake and growth rate functions in order to obtain more complex dynamics [Arino et al(2003)]. Another one is to use structured models. The population is then described by several variables such as the age or the size. But since it is difficult to identify the age of a cell, variables representing physiological states are often used to describe cell population [Lemesle and Gouzé(2005)]. Different models can be obtained by the choice of a structured variable: if this variable is continuous, a model consisting of partial differential equations is obtained; if this variable is discrete, the model results in a system of ordinary differential equations. In [Pascual and Caswell(1997)], the structured variable is continuous and represents the cell maturation along its cycle. Moreover, the cell number density is considered and the cell cycle is divided into two parts: in the first part, the cell needs external resources to maturate and in the second part, the cell does not need anything coming from the external environment. The authors [Pascual and Caswell(1997)] demonstrate using numerical simulations the existence of solutions exhibiting oscillations in cell number. In [Cazzador(1991)], a discrete variable representing two phases of the cell maturation (unbudded and budded cells) is chosen, the biomass concentrations are the variables, and numerical simulations show autonomous oscillatory behaviour.

We propose in the present paper two models inspired by both of the above models and an analytical proof of the oscillatory behavior for one of them. Our global aim is to obtain a simple unforced growth model, retaining the main qualitative biological hypotheses of the more complex model [Pascual and Caswell(1997)] and giving the same qualitative results (concerning the oscillations). 


\section{The chemostat}

The chemostat is a continuous device where microorganisms consume a nutrient to grow. This nutrient is provided by a constant inflow, and a blend of nutrient and of microorganisms is retrieved in the constant outflow. In this paper, we denote by $x$ the microorganisms concentration, $s$ the nutrient concentration, $V$ the volume of the chemostat, $s_{\text {in }}$ the limiting nutrient inflow, $q$ the constant flow passing through the chemostat and the dilution rate $d=\frac{q}{V}$. This description leads to the mass balance model :

$$
\left\{\begin{array}{l}
\dot{x}=\mu(s) x-d x \\
\dot{s}=-\alpha \mu(s) x-d s+d s_{i n}
\end{array}\right.
$$

where $\mu(s)$ is the specific growth rate function, $\alpha$ the growth yield coefficient. Different

kinds of functions exist in the literature; the Monod function $\mu(s)=\frac{\mu_{m} s}{s+k}$ is the most classical one. This classical model is not structured in a sense that it doesn't take into account the cell cycle stages. Its mathematical behavior is simple in the sense that every orbit converges towards the nontrivial equilibrium, if it exists.

\section{A partial differential equations oscillatory model}

In the literature, one of the most useful structured variables for describing cell growth is the size of the cell [Metz and Diekmann(1986)]. However, in the case of cell division, this variable is not specific enough since the size of a new born cell is difficult to determine. Therefore in [Pascual and Caswell(1997)] a new variable describing the position along the cell cycle is used. Moreover, to specify the cell division, the cell number density is considered. A dynamical system given by partial differential equations based on the Monod hypotheses is derived as follows:

$$
\begin{aligned}
& \frac{\partial x}{\partial t}+\frac{\nu_{0} s}{K+s} \frac{\partial x}{\partial p}=-(d+b(p)) x \text { for } p \in\left[p_{0} ; p_{c}\right] \\
& \frac{\partial x}{\partial t}+\nu_{c} \frac{\partial x}{\partial p}=-(d+b(p)) x \text { otherwise } \\
& \frac{d s}{d t}=d\left(s_{i n}-s\right)-\frac{V_{m} s}{K+s} x_{t o t}
\end{aligned}
$$

where $s$ is the substrate concentration, $x_{t o t}=\int_{p} x(t, p) d p$ the total population, $p$ gives the position along the cell cycle, $b(p)$ the division rate, $d$ the dilution rate, $s_{\text {in }}$ the inflow substrate concentration. The variable $p$ is normalized so that $p_{0}=0$ and the average cell divides at $p=1$. The cell cycle is divided into two parts: in the first part the cell needs external resources to grow and in the second part the cell does not need anything coming from the external environment. These two segments are separated by a transition point denoted $p_{c}$. Units of all the variables and parameters of this model can be found in [Pascual and Caswell(1997)].

Cell division is described by a boundary condition for the newborn cell at $p=0$

$$
\nu_{c} x(0, t)=2 \int_{p} b(p) x(p, t) d p
$$

where each cell divides into two immature cells. 
Proposition 1 (see [Pascual and Caswell(1997)]) For some parameters values (using the numerical method known as the escalator boxcar train [de Roos(1988)]), an oscillatory behavior is observed. The disappearance of oscillations depends on the value of $d$ or $s_{\text {in }}$.

Let us remark that some local mathematical results ensure the existence of exponentially periodic behavior [Webb(1995)] for such a structured model of cell population dynamics without cell division (the division rate could easily be incorporated into the analysis). Yet, the analysis of such models (especially the global analysis of non linear partial differential models) is often rather difficult or even intractable.

Since our purpose is to capture the essential qualitative mechanisms of the cell cycle, we want to build models easy to study analytically. To do this, an approach based on ordinary differential equations is followed and two structured model are built under the same main assumptions as the above system.

\title{
3. A non conservative size structured model
}

\subsection{The model}

Two main states are considered in our model. The first state corresponds to the immature phase and the second one to the mature one. In each state, the cell number density is considered: using this unit the division of the cell (meaning that one mature cell gives two immature cells) can be easily described.

The transfer rate between the two states is given by different maturation and division rates. The maturation rate depends on the limiting substrate, but the division rate does not. The uptake nutrient rate function is different from the maturation rate meaning that the energy used for growth is assumed to be different from the energy furnished by consumption of substrate. Thus the model does not have a conservative form. All these biological phenomena are described in Figure 1.

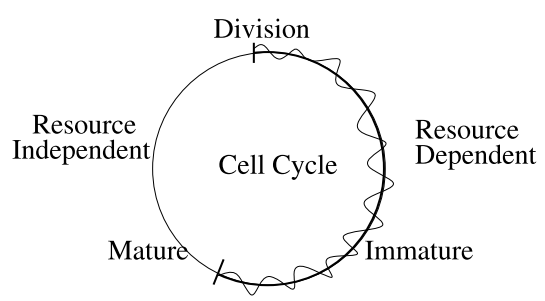

\author{
Division: \\ Mature $x_{2} \stackrel{k}{\longrightarrow} 2 x_{1}$ Immature \\ Maturation: \\ Immature $x_{1} \stackrel{g(s)}{\longrightarrow} x_{2}$ Mature \\ Nutrient uptake: \\ Substrate $s \stackrel{\nu(s)}{\longrightarrow}$ Mature $x_{2}+$ Immature $x_{1}$
}

Figure 1. (left) Two steps representing the cell cycle: substrate dependence and independence. (right) Biochemical diagram of the biological mechanisms: division at rate $k$, maturation at rate $g(s)$ and nutrient uptake at rate $\nu(s)$.

This leads to the model :

$$
\begin{array}{lc|l}
\dot{x_{1}}= & -g(s) x_{1}+2 k x_{2} & -d x_{1} \\
\dot{x_{2}}= & g(s) x_{1}-k x_{2} & -d x_{2} \\
\dot{s}= & -\nu(s)\left(x_{1}+x_{2}\right) & -d s+d s_{\text {in }} \\
& \text { Biological part } & \text { Physical part }
\end{array}
$$


where $s$ is the limiting substrate concentration (mass $/ l^{3}$ ), $x_{1}$ is the number density of the immature cells (number $/ l^{3}$ ), $x_{2}$ the number density of the mature cells (number $/ l^{3}$ ); $k>0$ is the division rate $(1 / T), g(s)$ the maturation rate $(1 / T), \nu(s)$ the uptake rate function (mass $/(T \times$ number $)) ; d$ is the dilution rate $(1 / T)$ and $s_{i n}$ the input substrate concentration $\left(\operatorname{mass} / l^{3}\right)$.

We choose initial conditions which have a biological meaning $x_{1}(0)>0, x_{2}(0)>0$ and $s \geq 0$.

As in the Monod model, some mathematical and qualitative hypotheses on the specific maturation rate $g(s)$ and the uptake rate $\nu(s)$ have to be made.

\section{Hypotheses H 3.1}

(a) $g(s) \geq 0$.

(b) $g(s)$ is an increasing function.

(c) There exists $\underline{s}$ and $\bar{s}$ such that $g^{\prime}(s) \gg 1$ for $\underline{s} \leq s \leq \bar{s}$.

(d) $g(s)$ is bounded.

(e) $g(0)=0$.

(f) $\nu(0)=0$ and $\nu($.$) is \mathcal{C}^{1}$, bounded, positive and increasing.

$(\mathrm{g})$ The domain of study of the system (2) is $\mathbb{R}_{+}^{3}$.

The function $g(s)$ which is assumed to be sigmoïdal (see Figure 2) can have the following form $g(s)=\frac{\alpha s^{a}}{k_{g}+s^{a}}$ with $a>1$. Let us remark that the unit of $\alpha$ is $1 / T$ and the unit of $k_{g}$ is the same as $s^{a}$. This formulation for the maturation rate means that cells need to have enough substrate to maturate, with a threshold effect. This is often observed in cell growth experiments (A. Sciandra, personal communication 2006). Moreover, the fact that the slope $g^{\prime}(s) \gg 1$ means that we are free to choose the slope as large as desired: this feature will be useful in the convergence proofs. The function $\nu(s)$ can have the following form $\nu(s)=\frac{\nu_{m} s}{k_{n}+s}$ where the unit of $\nu_{m}$ is mass $/(T \times$ number $)$ and the unit of $k_{n}$ is the same as $s$.

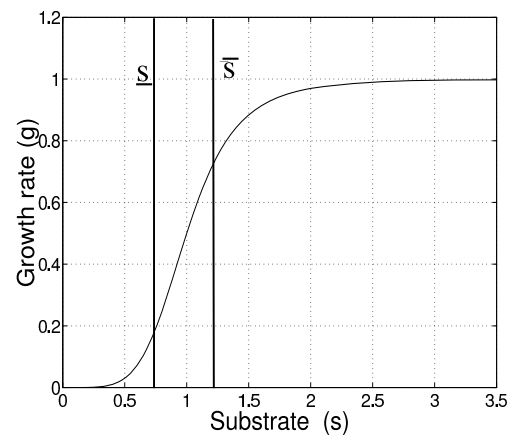

Figure 2. Maturation rate function.

We add two useful hypotheses: 
Hypothesis H $3.2 k-d>0$ and $\frac{d(k+d)}{k-d}<g\left(s_{i n}\right)$.

Hypothesis H $3.3 \underline{s}<s^{*}<\bar{s}$

The first hypothesis ensures the existence and the uniqueness of a non-trivial equilibrium point. The second one implies that $g^{\prime}\left(s^{*}\right) \gg 1$, meaning that the slope of the maturation function is very stiff at equilibrium.

\subsection{Existence of steady states, boundedness and an invariant set for model (2)}

The set of study of the system (2) is $\mathbb{R}_{+}^{3}$ since it is of biological interest.

Proposition 2 Under (H3.2) two steady states exist for the system (2): (0, 0, $\left.s_{\text {in }}\right)$ referred to as the washout point (i.e. whole population disappearance), $\left(x_{1}^{*}, x_{2}^{*}, s^{*}\right)$ referred to as the non trivial point (i.e. population persists).

Proof: Computing the steady states, we find that :

- $\left(0,0, s_{i n}\right)$ is always a solution.

- a positive equilibrium $\left(x_{1}^{*}, x_{2}^{*}, s^{*}\right)$ such that $x_{1}^{*}>0, x_{2}^{*}>0, s^{*}>0$ exists and is unique. Indeed,

$g\left(s^{*}\right)=\frac{d(k+d)}{k-d}$ has a unique solution under the hypothesis (H3.2) and because $g$ is strictly increasing.

Let us remark that $g\left(s_{i n}\right)>g\left(s^{*}\right)$, and since $g$ is strictly increasing $s_{i n}>s^{*}$.

Proposition 3 Under the hypotheses (H3.1), the closed domain of study $\mathbb{R}_{+}^{3}$ is invariant for (2).

Proof: Computing the dynamical equations $\dot{x}_{1}, \dot{x}_{2}$ and $\dot{s}$ at $x_{1}=0, x_{2}=0$ and $s=0$ respectively and showing that the edges are repulsive, we can prove the invariance of the set $\mathbb{R}_{+}^{3}$ for the system (2).

Remark 1 Consider the subset $U=\mathbb{R}_{+}^{2} \times\left[0, s_{i n}\right]$. We can prove that this set is invariant for the system (2). Indeed, computing the dynamical equation $\dot{s}$ at $s_{i n}$, we obtain $\dot{s}_{\left(s=s_{i n}\right)}=$ $-\nu\left(s_{i n}\right)\left(x_{1}+x_{2}\right)<0$.

Proposition 4 In the domain $\mathbb{R}_{+}^{3}$, the state variables are bounded.

Proof: Let $c>0$ be defined such that for all $s \geq 0, c>\frac{g(s)}{\nu(s)}$ (see Remark 2). Let $V=x_{1}+2 x_{2}+c s$ be a positive definite function. Then we obtain:

$$
\dot{V}=-d V+(g(s)-c \nu(s)) x_{1}-\nu(s) x_{2}+d c s_{i n}<-d V+d c s_{i n}
$$

so that

$$
V(t)<\left(V(0)-c s_{i n}\right) e^{-d t}+c s_{i n}
$$

Then for $V(0)>c s_{i n}, V(t)<V(0)$ and for $V(0) \leq c s_{i n}, V(t) \leq c s_{i n}$. Thus the variables $x_{1}, x_{2}, s$ are bounded. 
Remark 2 The existence of a positive constant $c$ is always possible even near $s=0$. Indeed since $g(s)$ is a sigmoïdal function and $\nu(s)$ a Monod like function, near $0, g(s) \sim$ $k s^{a}$ and $\nu(s) \sim k^{\prime} s$ with $k>0, k^{\prime}>0, a>1$. Thus $\frac{g(s)}{\nu(s)}$ exists on $\mathbb{R}_{+}$and can be bounded.

Using the hypothesis (H3.2), we prove that, for the washout point $\left(0,0, s_{\text {in }}\right)$. one eigenvalue is positive and the two others are negative. We can conclude that the washout point is unstable. Moreover, the following proposition can be used to prove persistence of the positive solutions (cf [Lemesle and Gouzé(2008)] for more details).

Proposition 5 The only eigenvector intersecting $\mathbb{R}_{+}^{3}$ is the vector associated with the positive eigenvalue. The stable manifold of the washout equilibrium does not intersect the positive orthant.

\subsection{Global analysis}

To study the stability of the non trivial point when it exists, we make the change of variables $z=\ln \left(x_{1}+x_{2}\right), u=\frac{x_{1}}{x_{1}+x_{2}}, s=s$. Because of the dissipativity and the uniform persistence of the original system, the new system is well defined in the open set $D_{1}=\mathbb{R} \times \mathbb{R}_{+}^{*} \times \mathbb{R}_{+}^{*}$. Moreover, there exists a compact subset $B \in D_{1}$ which attracts all solutions starting in $D_{1}$. From a biological point of view, we remark that this change of variables amounts to taking the total number of cells, and the proportion of mature cells, as the new variables.

We obtain the new dynamical system:

$$
\begin{aligned}
& \dot{z}=k(1-u)-d \\
& \dot{u}=-g(s) u+k(1-u)(2-u) \\
& \dot{s}=-\nu(s) e^{z}-d s+d s_{i n}
\end{aligned}
$$

with its associated Jacobian matrix

$$
J=\left(\begin{array}{ccc}
0 & -k & 0 \\
0 & -g(s)-k-2 k(1-u) & -g^{\prime}(s) u \\
-\nu(s) e^{z} & 0 & -\nu^{\prime}(s) e^{z}-d
\end{array}\right)
$$

This new system is competitive in $D_{1}$ since all the off-diagonal terms of the Jacobian matrix are non positive [Smith(1995)]. Moreover this system is irreducible in $D_{1}$ since $J$ is an irreducible matrix.

Proposition 6 Under the hypothesis (H3.3), the non trivial equilibrium $\left(z^{*}, u^{*}, s^{*}\right)$ is unstable. Moreover, the stable manifold is one-dimensional.

cf [Lemesle and Gouzé(2008)].

To prove the existence of a stable limit cycle, the following theorem [Zhu and Smith(1994)] using competitive property is applied:

Theorem 1 (see [Zhu and Smith(1994)]) Let $\dot{x}=f(x)$ be a dissipative, irreducible and competitive system in an open subset $D \subset \mathbb{R}^{3}$. Moreover, let $D$ be a $p$-convex system of $\mathbb{R}^{3}$. Assume that $D$ contains a unique equilibrium point $\xi^{*}$ and $\operatorname{det}\left(J^{*}\right)<0$. Then either 
(i) $\xi^{*}$ is stable, or

(ii) there exists a non trivial orbitally stable periodic orbit in D.

Let us denote the non trivial steady state $\left(x_{1}^{*}, x_{2}^{*}, s^{*}\right)=\xi$ and consider (3) in the open set $D_{1}$.

Proposition 7 Under the hypotheses (H3.2), a non trivial stable periodic orbit exists for (3) in $D_{1}$.

Proof: To prove this proposition, let us verify the hypotheses of the above theorem 1. We compute the Jacobian matrix $J$ at $\xi$.

(a) The system is dissipative, irreducible and competitive using proposition 4.

(b) $D_{1}$ is $p$-convex by definition and contains a unique equilibrium point $\xi^{*}$.

$(c)$ Since $J^{*}$ has two non negative eigenvalues, $\xi^{*}$ is unstable and moreover $\operatorname{det}\left(J^{*}\right)<$ 0 (see proof of proposition 6).

Thus since all the hypotheses of theorem 1 are verified, a non trivial stable positive orbit exists for (3), and therefore for system (2).

Remark 3 (The dilution rate as a bifurcation parameter) An important hypothesis ensuring the existence of the limit cycle is $g^{\prime}\left(s^{*}\right) \gg 1$. Recall that the non trivial point is defined such that:

$$
g\left(s^{*}\right)=\frac{d(k+d)}{k-d}=f(d)
$$

where $f(d)$ is an increasing function of $d$. When the dilution rate $d$ varies, $s^{*}$ can leave the interval $[\underline{s}, \bar{s}]$ and $g^{\prime}\left(s^{*}\right)$ may become smaller (see Figure 3). Thus for higher or lower dilution rates, the limit cycle may disappear and the non trivial equilibrium becomes stable or disappears.

\subsection{Simulations}

We choose for parameter values $k=0.6$ days $^{-1}, s_{\text {in }}=10 \mu \mathrm{mol} \times \mathrm{L}^{-1}$,

$$
g(s)=\frac{s^{18}}{1+s^{18}}\left(\alpha=1 \text { days }^{-1}, k_{g}=1 \mu \mathrm{mol} \times \mathrm{L}^{-1}\right) \text { and } \nu(s)=\frac{s}{1+s}\left(\nu_{m}=\right.
$$

$1 \mu \mathrm{mol} \times 10^{6}$ cells $\times$ days $\left.^{-1}, k_{n}=1 \mu \mathrm{mol} \times \mathrm{L}^{-1}\right)$, which verify $(H 3.3)$ for $d=0.02$ days $^{-1}$, 0.1 days $^{-1}$ and 0.25 days $^{-1}$. These values correspond to classical common values usually taken in growth phytoplankton chemostat experiments (A. Sciandra, private communications). Notice that the exponent 18 put in the formula of $g(s)$ has to be large so that all the mathematical hypotheses are verified. Notice also that the unit of $s$ is $\mu \mathrm{mol} \times \mathrm{L}^{-1}$ and the unit of $x_{1}$ and $x_{2}$ is $10^{6}$ cells $\times \mathrm{L}^{-1}$.

A limit cycle can be observed for $d=0.1$ days $^{-1}$ which disappears for higher dilution values. Indeed, an approximate study of the function $g(s)$ and its derivative provide $s \sim$ $0.9 \mu \mathrm{mol} \times \mathrm{L}^{-1}, \bar{s} \sim 1 \mu \mathrm{mol} \times \mathrm{L}^{-1}$; then $g(0.9)=0.07$ days $^{-1}, g(1)=0.5$ days $^{-1}$ and therefore if $0.07 \gtrsim f(d) \gtrsim 0.5$, there is no limit cycle. With the chosen values we have $f(0.02) \sim 0.02, f(0.1) \sim 0.14$ and $f(0.25) \sim 0.6$. Thus for $d=0.02$ days $^{-1}$ and $d=0.25$ days $^{-1}$, the limit cycle disappears (see Figure 3 ). 

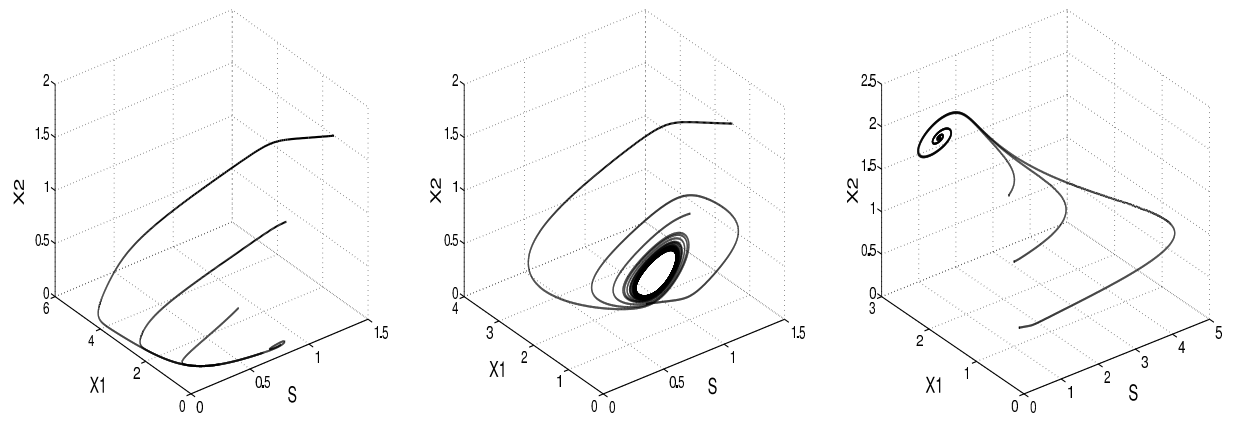

Figure 3. Phase diagram of the model given by equation (2) illustrating disappearance of the limit cycle for different values of $d\left(d=0.02\right.$ days $^{-1}, d=0.1$ days $^{-1}, d=0.25$ days $^{-1}$ )

\section{A conservative size structured model}

As in the previous section, we consider two stages in the cell cycle. But the variables of this model are the biomasses, and not the cell number density as above.

\subsection{Description of the model}

The model is therefore similar to the first model, but has to check mass balance constraints: a mature cell does not give two immature cells (as above), but the biomass of the mature cells results in a larger biomass of immature cells, with consumption of substrate.

That leads to the dynamical system :

$$
\left\{\begin{array}{l}
\dot{x_{1}}=-\mu_{1}(s) x_{1}+(1+\beta) \mu_{2}(s) x_{2}-d x_{1} \\
\dot{x_{2}}=(1+\alpha) \mu_{1}(s) x_{1}-\mu_{2}(s) x_{2}-d x_{2} \\
\dot{s}=-\alpha \mu_{1}(s) x_{1}-\beta \mu_{2}(s) x_{2}-d s+d s_{i n}
\end{array}\right.
$$

where $x_{1}$ is the biomass concentration of the immature cells, $x_{2}$ the biomass concentration of the mature cells; $\mu_{1}(s)$ is the growth rate function, $\mu_{2}(s)$ the division rate function; $\alpha \mu_{1}(s), \beta \mu_{2}(s)$ the intake rates of $x_{1}$ and $x_{2}$, respectively.

Hypotheses $\mathbf{H} 4.1 \mu_{1}(s) \neq \mu_{2}(s)$

$\mu_{1}(s)$ and $\mu_{2}(s)$ positive increasing $\mathcal{C}^{1}$ functions with $\mu_{1}(0)=\mu_{2}(0)=0$.

\subsection{Study of the model}

Two steady states exist for this model: the washout steady state $x_{1}=0, x_{2}=0, s=s_{\text {in }}$ and the normal steady state $x_{1}^{*}>0, x_{2}^{*}>0,0<s^{*}<s_{\text {in }}$.

Proposition 1 The system (4) has no periodic orbit.

First, we prove the following lemma.

Lemma 1 The positive orthant $\mathbb{R}^{3+}$ is invariant for the system (4). 
Sketch of the proof. The ideas are the same as before. First we prove the repulsivity of the faces of the orthant $\mathbb{R}^{3+}$, without $\left(0,0, s_{i n}\right)$. Then, we prove the instability of the washout steady state considering the evaluated Jacobian matrix :

$$
J_{w o}=\left(\begin{array}{llr}
-\mu_{1}\left(s_{i n}\right)-d & (1+\beta) \mu_{2}\left(s_{i n}\right) & 0 \\
(1+\alpha) \mu_{1}\left(s_{i n}\right) & -\mu_{2}\left(s_{i n}\right)-d & 0 \\
-\alpha \mu_{1}\left(s_{i n}\right) & -\beta \mu_{2}\left(s_{i n}\right) & -d
\end{array}\right)
$$

and using the hypotheses 4.1 and the existence of the normal steady state $\left(d^{2}+\right.$ $\left(\mu_{1}\left(s^{*}\right)+\mu_{2}\left(s^{*}\right)\right) d-\mu_{1}\left(s^{*}\right) \mu_{2}\left(s^{*}\right)(\alpha+\beta+\alpha \beta)=0$ and $\mu_{i}\left(s^{*}\right)<\mu_{i}\left(s_{\text {in }}\right)$ for $\left.i=1,2\right)$.

Moreover, we need the lemma:

Lemma 2 The mass conservation principle is verified: $x_{1}+x_{2}+s$ tends to $s_{\text {in }}$ when $t$ tends to infinity.

Proof. Let us consider the variable $z=x_{1}+x_{2}+s$. Then we obtain the equation : $\dot{z}=-d z+d s_{\text {in }}$. Thus, we get the result.

As we want to study the asymptotic behavior of the system, only two variables are needed to describe the model using the above lemma. Then, we can apply the Bendixson criterion.

Theorem 2 If on a simply connected region $D \subseteq \mathbb{R}^{2}$ the trace of the associated Jacobian matrix is not identically zero and does not change sign, then the dynamical system has not closed orbit lying entirely in $D$.

Proof See [Guckenheimer(1983)].

Thus, we study the sub-system in $\mathbb{R}^{2}$ :

$$
\left\{\begin{array}{l}
\dot{x_{2}}=(1+\alpha) \mu_{1}(s)\left(s_{i n}-s-x_{2}\right)-\mu_{2}(s) x_{2}-d x_{2}=f_{1}\left(x_{2}, s\right) \\
\dot{s}=-\alpha \mu_{1}(s)\left(s_{i n}-s-x_{2}\right)-\beta \mu_{2}(s) x_{2}-d s+d s_{i n}=f_{2}\left(x_{2}, s\right)
\end{array}\right.
$$

with the associated Jacobian matrix

$$
J=\left(\begin{array}{cc}
-(1+\alpha) \mu_{1}(s)-\mu_{2}(s)-d & -(1+\alpha) \mu_{1}(s)+(1+\alpha) \mu_{1}^{\prime}(s)\left(s_{i n}-s-x_{2}\right) \\
\alpha \mu_{1}(s)-\beta \mu_{2}(s) & \alpha \mu_{1}(s)-\alpha \mu_{1}^{\prime}(s)\left(s_{i n}-s-x_{2}\right)-\beta \mu_{2}^{\prime}(s) x_{2}-d
\end{array}\right)
$$

As the trace is negative and does not change sign in a simply connected region, we can conclude that there is no closed orbit for the system (4).

\section{Comments and Conclusion}

These two simple ODE models are based on the same biological hypotheses as the PDE model [Pascual and Caswell(1997)]. Indeed, these PDE models have a richest behavior but their study is difficult. Thus, the simplicity of the proposed models and their easy mathematical study are advantages. These two kind of models which describe the structure 
in two stages, the growth and the transfer between the two stages, do not give similar results. The first one (the variables are the cell number density) has an oscillatory behaviour, quite similar to the model of [Pascual and Caswell(1997)]. The second model is built with the cell biomass, and the oscillatory behaviour is precluded by the mass conservation. It would be interesting to study if such a model with more stages (at least three) could oscillate.

\section{References}

[Arino et al(2003)] Arino, J., Pilyugin, S and Wolkowicz, G. 2003. Considerations on yield, cellular growth, and competition in chemostat models. Canadian Applied Mathematics Quarterly 11, 107141.

[Cazzador(1991)] Cazzador, L. 1991. Analysis of oscillations in yeast continuous cultures by a new simplified model. Bulletin of Mathematical Biology 53, 685-700.

[Guckenheimer(1983)] Guckenheimer, J. and Holmes, P., 1983. Nonlinear Oscillations, Dynamical Systems, and Bifurcations of Vector Fields, Springer-Verlag.

[Lemesle and Gouzé(2005)] Lemesle, V. and Gouzé J-L. 2005. A biochemically based structured model for phytoplankton growth in the chemostat. Ecological Complexity 2, 21-33.

[Lemesle(2004)] Lemesle, V. 2004. Modélisation structurée de la croissance cellulaire en chemostat: analyse et estimation. $\mathrm{PhD}$ Thesis Nice-Sophia Antipolis University.http://tel.ccsd.cnrs.fr/documents/archives0/00/00/65/02/

[Lemesle and Gouzé(2008)] Lemesle V. and Gouzé J.-L. 2008. A simple unforced oscillatory growth model in the chemostat. Bulletin of Mathematical Biology 70, 2, 344-357.

[Metz and Diekmann(1986)] Metz, J.A.J. and Diekmann, O. 1986. The Dynamics of Physiologically Structured Populations. Springer-Verlag, New York.

[Monod(1942)] Monod, J. 1942. Recherches sur la Croissance des Cultures Bactériennes. Herman, Paris.

[Nisbet and Gurney(1982)] Nisbet, R., Gurney, W. 1982. Modelling Fluctuating Populations. John Wiley and Sons, New York.

[Pascual and Caswell(1997)] Pascual, M., Caswell, H. 1997. From the Cell Cycle to Population Cycles in Phytoplankton-Nutrient Interactions. Ecology 3, 897-912.

[Porro et al.(1988)] Porro, D., Martegani, E., Ranzi, B.M., Alberghina, L. 1988. Oscillations in continuous cultures of budding yeast: a segregated parameter analysis. Biotechnology and Bioengineering 32, 411-417.

[de Roos(1988)] de Roos, A.M. 1988. Numerical methods for structured population: the escalator boxcar train. Numerical Methods for Partial Differential Equations 4, 173-195.

[Sciandra et al.(2003)] Sciandra A., Harlay J., Lefèvre D., Lemée R., Rimmelin P., Denis M., Gattuso J.-P. 2003. Response of coccolithophorid Emiliania huxleyi to elevated partial pressure of CO2 under nitrogen limitation. Mar. Ecol. Prog. Ser. 261: 111-122.

[Smith and Waltman(1995)] Smith, H. and Waltman, P. 1995. The theory of the chemostat. Cambridge University Press.

[Smith(1995)] Smith, H. 1995. Monotone Dynamical Systems. An Introduction to the theory of competitive and cooperative systems. American Mathematical Society.

[Webb(1995)] Webb, G.F. 1995. Periodic and chaotic behavior in structured models of cell population dynamics. Pitman Research Notes on Mathematical Series 324, 40-49.

[Zhu and Smith(1994)] Zhu, H-R. and Smith, H. 1994. Stable periodic orbits for a class of three dimensional competitive systems. Journal of Differential Equations 110, 143-156. 